\title{
Comportamento anoréxico e percepção corporal em universitários
}

\author{
Anorexic behavior and body self-perception on universitarians \\ Tatiane Dutra Gonçalves', Mariana Prado Barbosa', Luiz Carlos Laureano da Rosa', Alexandra Magna Rodrigues'
}

\section{RESUMO}

Objetivo: Avaliar a percepção corporal e a prevalência de sintomas de anorexia nervosa em estudantes universitários. Métodos: Estudo transversal, no qual a seleção da amostra foi realizada por conveniência. Aplicou-se o questionário teste de atitudes alimentares (EAT- 26) e teste de imagem corporal, para avaliar os sintomas de anorexia nervosa e a percepção corporal, respectivamente. Para a análise estatística dos dados utilizou-se o teste binomial para verificar a existência de associação entre as variáveis qualitativas. Resultados: Participaram do estudo 149 estudantes de nutrição e 78 estudantes de educação física. Observou-se que 10,3\% e 14,1\% dos estudantes de educação física e nutrição, respectivamente, apresentaram fatores de risco para desenvolver anorexia nervosa e o teste de imagem corporal evidenciou proporções elevadas de insatisfação com a forma corporal, em ambos os cursos: 75,8\% e 78,2\% para os cursos de nutrição e educação física, respectivamente. Conclusão: Os estudantes de nutrição mostraram maior tendência de apresentar comportamento de risco para anorexia nervosa, com predominância no sexo feminino. Além disso, os resultados evidenciaram proporções elevadas de insatisfação com a imagem corporal, em ambos os cursos. Estes resultados são preocupantes, tendo em vista que são futuros profissionais que possuem papel fundamental na detecção e, no caso do nutricionista, no manejo destes distúrbios.

\section{Palavras-chave \\ Universitários, anorexia \\ nervosa, imagem corporal.}

\section{Keywords}

College students, nervous anorexy, corporal body image

\section{ABSTRACT}

Objective: To assess body perception and prevalence of symptoms of nervous anorexy in college students. Methods: Cross-sectional study with convenience sample. We used the Eating Attitudes Test questionnaire (EAT-26) and the Body Image Test to evaluate symptoms of nervous anorexy and the body perception on the participants, respectively. In the statistical analysis, Binomial test was used to assess the association between qualitative variables. Results: This study included 149 nutrition and 78 physical education students. The results showed that 10.3\% and $14.1 \%$ of the physical education and nutrition students, respectively, presented risk factors for the development of anorexy, and the Body Image Test revealed high levels of dissatisfaction regarding body shape of students in both courses: $75.8 \%$ and $78.2 \%$ in nutrition and physical education, respectively. Conclusion: Students of nutrition showed greater tendency to present risk behavior for developing nervous anorexy, predominantly in females. Moreover, the results showed high proportions of dissatisfaction with body image in both courses. These results raise concern, since the physical education teachers and nutritionists are the professionals who play a key role in detecting and, in the case of a nutritionist, managing these disorders.

1 Departamento de Psiquiatria da Universidade de Taubaté (UNITAU). 
Os transtornos alimentares (TA) são entidades de grande importância médico-social, pois podem comprometer seriamente a saúde dos indivíduos sintomáticos. Atitudes de comportamento alimentar preocupantes e problemáticas relativas ao medo da "gordura" são comumente encontradas. Por causa de sua importância epidemiológica, urge ampliar esses estudos com a utilização de instrumentos específicos para seu rastreamento, para que o processo de intervenção e prevenção na população sejam efetivos’

Os distúrbios alimentares ocorrem mais freqüentemente no sexo feminino². Os homens também são acometidos, mas em proporções menores, representando apenas 10\% dos casos dos TA'.

A anorexia nervosa (AN) é um transtorno alimentar representada pela distorção na maneira como o indivíduo avalia a forma, o peso e o tamanho de seu corpo (imagem corporal). Somado à distorção de imagem corporal, há medo mórbido de engordar e recusa alimentar. Para perder peso, o indivíduo submete-se a longos períodos de jejum ou restrição alimentar. Uso de inibidores de apetite, laxantes e diuréticos e atividade física também podem ocorrer. Outro sinal importante no sexo feminino é a presença de amenorréia ou diminuição da libido no sexo masculino ${ }^{3}$.

A AN tem complicações sérias associadas com a desnutrição, como comprometimento cardiovascular, desidratação, distúrbios eletrolíticos, distúrbios na motilidade gastrintestinal, infertilidade, hipotermia e outras evidências de hipometabolismo ${ }^{4}$. E constitui, hoje, "epidemia silenciosa", talvez por isso, ainda não seja reconhecida pelas políticas públicas no setor da saúde ${ }^{5}$.

Os TA têm etiologia multifatorial, ou seja, são determinados pela diversidade de fatores que interagem entre si de modo complexo, para produzir e, muitas vezes, perpetuar a doença. Classicamente, distinguem-se os fatores predisponentes, precipitantes e os mantenedores dos TA ${ }^{6}$.

O sexo feminino, de maneira geral, é muito vulnerável à aceitação das pressões sociais, econômicas e cultural associada aos padrões estéticos. A sociedade rejeita, discrimina e reprova pessoas obesas².

O modelo de beleza imposto pela sociedade atual corresponde ao corpo magro, não levando em consideração os aspectos relacionados com a saúde e as diferentes constituições físicas da população. Esse padrão distorcido de beleza acarreta número cada vez maior de mulheres que se submetem a dietas para controle de peso, ao excesso de exercícios físicos e ao uso indiscriminado de laxantes, diuréticos e drogas anorexígenas?.

O ideal do corpo perfeito preconizado pela nossa sociedade e veiculado pela mídia leva as mulheres à insatisfação crônica com seu corpo, culpando-se, muitas vezes, por alguns quilos a mais, ou adotando dietas altamente restritivas e exercícios físicos extenuantes como forma de compensar as calorias ingeridas em excesso, na tentativa de corresponder ao modelo cultural vigente ${ }^{5}$.

Em geral, os homens estão mais satisfeitos com seu corpo e os percebem com menos distorção ${ }^{8}$. A maioria considera o corpo mais musculoso como representação da imagem corporal masculina ideal ${ }^{9}$. Ao contrário das mulheres, que estão mais preocupadas com o peso, os homens relatam maior preocupação com a forma física e a massa corporal, e buscam, especificamente, aumento da massa muscular, por isso fazem mais exercícios físicos ${ }^{8}$.

A relação entre profissionais do sexo feminino (que exigem baixo peso corporal) e os TA tem sido discutida na literatura. Entretanto, a literatura é escassa em relação aos TA em indivíduos do sexo masculino. Alguns grupos apresentam maiores chances de desenvolver TA, destacando-se aqueles cujas atuações estão ligadas à preocupação exagerada com o peso ou a forma corporal - atletas, modelos, estudantes de nutrição ${ }^{2,8}$.

Tendo em vista o exposto, o objetivo do presente estudo foi avaliar a percepção corporal e a prevalência de sintomas de AN em estudantes de nutrição e educação física de uma universidade do Vale do Paraíba, SP.

\section{MÉTODOS}

A amostra do estudo foi selecionada por conveniência. Foram avaliados os estudantes dos cursos de nutrição e educação física de uma universidade de Taubaté, de ambos os sexos, da $1^{\mathrm{a}}$ a $3^{\mathrm{a}}$ série (nutrição) e da $2^{\mathrm{a}}$ a $4^{\mathrm{a}}$ série (educação física), que foram agrupados da seguinte forma: turma 1 ( $1^{\circ}$ e $2^{\circ}$ ano), turma 2 ( $2^{\circ}$ e $3^{\circ}$ ano) e turma 3 ( $3^{\circ}$ e $4^{\circ}$ ano) de nutrição e educação física, respectivamente.

Para avaliar a prevalência de sintomas de AN utilizou-se o Eating Attitudes Test (EAT-26), elaborado por Garner et al.10 e traduzido para o português por Nunes et al., em 1994"1. É um questionário de autopreenchimento, constituído de 26 questões com seis opções de resposta: sempre, muito freqüente, freqüentemente, às vezes, raramente e nunca. O teste é muito utilizado em estudos epidemiológicos para rastrear indivíduos supostamente suscetíveis ao desenvolvimento de distúrbio da conduta alimentar e pessoas cujas respostas ao teste atingem escore igual ou superior a 20. O cálculo do escore foi realizado por meio de uma escala para cada questão, cuja pontuação é a seguinte: sempre (3 pontos); muito freqüente (2 pontos); freqüente (1 ponto) e às vezes/raramente/nunca (0).

Aplicado o instrumento, os escores obtidos em cada questão do EAT-26 são somados e computados para cada pessoa avaliada. Caso o total de escores encontrado seja 
maior ou igual a 20, o EAT-26 é considerado positivo e confirmada a presença de atitudes alimentares patológicas e risco para o desenvolvimento de $\mathrm{AN}$; e escore entre 0 a 19 considerou-se fora de risco.

Para avaliar a percepção corporal adotou-se o teste de imagem corporal ${ }^{12}$, que consiste de nove figuras, divididas em quatro categorias: silhueta 1: delgado (magro); silhuetas 2 a 5: normal; silhuetas 6 e 7: sobrepeso e silhuetas 8 e 9: obeso.

As figuras esquemáticas foram apresentadas individualmente a cada estudante e solicitada a escolha de uma entre essas figuras, aquela que se pareça mais com o seu corpo (auto-imagem corporal), e, em seguida, solicitou-se que assinalasse a figura que gostaria de ser (desejo).

Também foram coletados dados sobre idade e sexo, peso $(\mathrm{kg})$ e estatura $(\mathrm{m})$ referidos pelos participantes.

A análise estatística foi realizada por meio do programa Bio Estat e utilizou-se o teste binomial para verificar a existência de associação entre as variáveis qualitativas, considerando-se como significante o valor de $p \leq 0,05$.

Esta pesquisa foi aprovada pelos comitês de ética da Universidade de Taubaté, e todos os indivíduos assinaram termo de consentimento e esclarecido antes de qualquer procedimento do estudo fosse iniciado.

\section{RESULTADOS}

A população estudada constituiu-se de 227 estudantes universitários, de ambos os sexos, sendo: 149 alunos do curso de nutrição com idade média de 22,57 anos e 78 alunos do curso de educação física com idade média de 23,65 anos.

De acordo com os resultados, observou-se que $14,1 \%$ e 10,3\% dos estudantes de nutrição e educação física, respectivamente, apresentaram sintomas de AN ( $p>0,05)$.

Houve tendência dos estudantes do sexo feminino apresentarem comportamento de risco para AN mais elevado, quando comparado aos do sexo masculino, em ambos os cursos ( $p>0,05)$ (Figura 1).

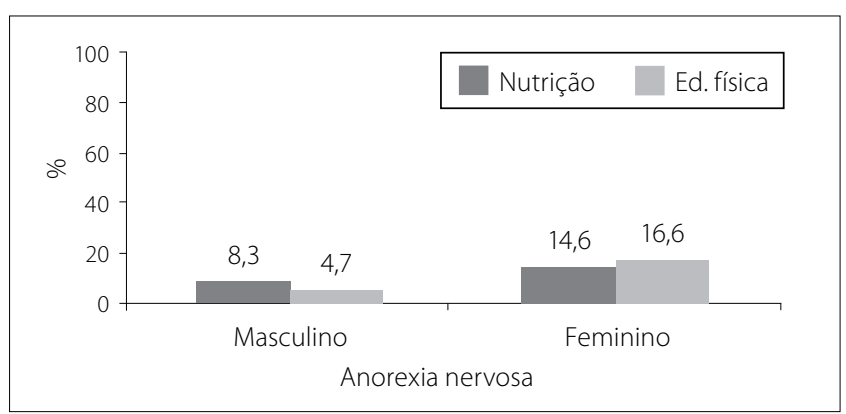

$p>0,05$, segundo teste binomial.

Figura 1. Sintomas de anorexia nervosa em alunos de nutrição e de educação física, segundo o gênero.
A Figura 2 demonstra que a proporção de estudantes com sintomas de AN apresentou-se maior na turma 1 de nutrição em relação a turma 1 de educação física $(p<0,05)$. Entretanto, ressalta-se que entre as demais turmas não houve diferença estatisticamente significante $(p>0,05)$.

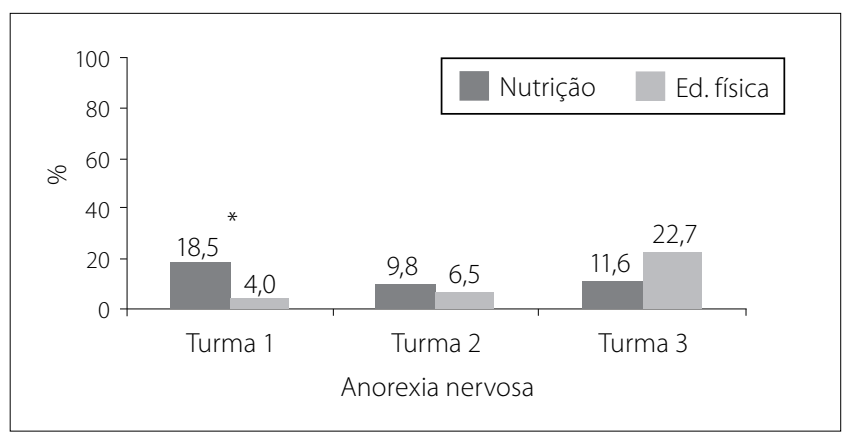

$p<0,05$, segundo teste binomial.

Figura 2. Sintomas de anorexia nervosa em alunos de nutrição e educação física, por turma.

Ao avaliar a percepção corporal observou-se que 75,8\% dos estudantes de nutrição e 78,2\% de educação física encontravam-se insatisfeitos com a forma corporal $(p>0,05)$. Verificou-se, ainda, diferença entre insatisfação corporal entre os alunos dos sexos masculino e feminino $(p<0,05)$ (Figura 3).

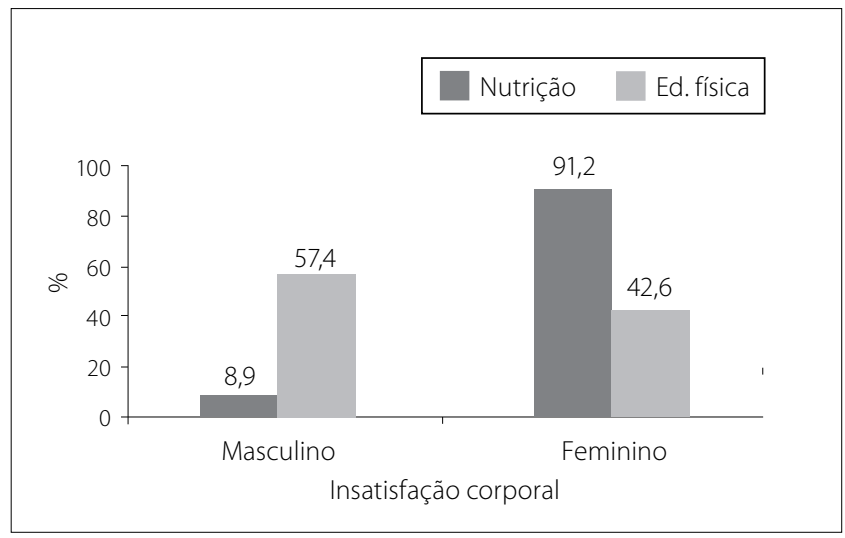

$p<0,05$, segundo teste binomial.

Figura 3. Distribuição percentual quanto à percepção corporal dos estudantes, segundo o gênero.

De acordo com o teste de imagem corporal, a maioria dos alunos de nutrição e educação física se "sentia" com excesso de peso (Figura 4).

A Figura 5 mostra que a proporção de alunos do curso de nutrição que desejavam ser normais (eutróficos) foi maior do que no grupo de educação física, que apresentaram maior desejo em aumentar de peso $(p<0,05)$. 


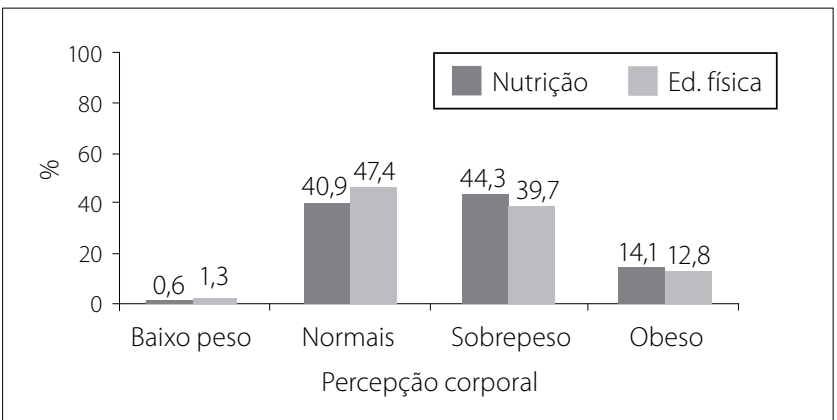

$p>0,05$, segundo teste binomial.

Figura 4. Distribuição percentual quanto à percepção corporal dos estudantes.

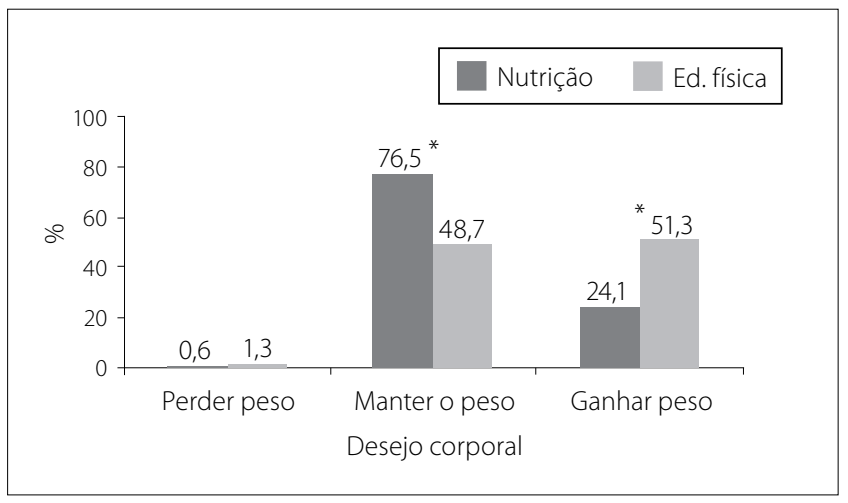

${ }^{*} p<0,05$, segundo teste binomial.

Figura 5. Distribuição percentual quanto ao desejo corporal dos estudantes.

A Tabela 1 mostra a relação entre a insatisfação corporal e a presença de sintomas de AN.

Tabela 1. Relação entre insatisfação corporal e a presença de sintomas de anorexia nervosa.

\begin{tabular}{lccccc}
\hline \multirow{2}{*}{ Sintomas de anorexia nervosa } & \multicolumn{4}{c}{ Insatisfação corporal } \\
\cline { 2 - 5 } & \multicolumn{3}{c}{ Nutriçăa } & \multicolumn{3}{c}{ Educaçăo física } \\
\cline { 2 - 5 } & Sim & Não & Sim & Não \\
\hline Não & 20 & 1 & 8 & 0 \\
\hline
\end{tabular}

$p>0,05$, segundo teste binomial.

\section{DISCUSSÃO}

Os TA são cada vez mais focos da atenção dos profissionais da área da saúde por apresentarem significativos graus de morbimortalidade ${ }^{13}$. Vitolo et al. ${ }^{14}$, verificaram que a população universitária pode ser foco de estudos que propiciem maiores esclarecimentos sobre os TA.

A taxa de prevalência de AN é em torno de $1 \%$ na população geral, com 90\% dos casos em mulheres ${ }^{3}$. E seu diagnóstico exige entrevista clínica, tornando mais difícil avaliar grupos. Por esse motivo, questionários que identifique sintomas têm sido utilizados para localizar grupos de risco para o desenvolvimento de $\mathrm{AN}^{\prime \prime}$.

De acordo com estudo realizado no Rio de Janeiro, com estudantes de nutrição do sexo feminino, 14\% das alunas apresentaram sintomas de $\mathrm{AN}^{15}$. Este estudo encontrou resultados semelhantes ao observado no presente, no que se refere a estudantes do sexo feminino de nutrição $(14,6 \%)$.

Conforme estudo de Furlann', que avaliou 45 alunas de educação física de uma universidade de Sorocaba/SP, nenhuma apresentou sintomas de AN. Estes resultados divergem dos encontrados no presente estudo que verificou que 16,6\% das alunas do curso de educação física apresentavam comportamento e risco para a AN.

O presente trabalho mostrou que o sexo feminino apresentou maiores proporções de sintomas de $\mathrm{AN}$, em ambos os cursos, concordando com os dados encontrados na literatura ${ }^{15}$.

Consoante a Sociedade Brasileira de Psiquiatria Clínica (SBPC), a cada ano, aumenta o número de pessoas que desenvolve graves transtornos do comportamento alimentar. A maior parte, mais de $90 \%$, é composta de adolescentes e mulheres jovens ${ }^{17}$. Os TA são bem menos freqüentes nos homens, por essa razão, o universo masculino tem sido relativamente negligenciado e ignorado pelos especialistas ${ }^{18}$.

Atualmente as descrições de TA no sexo masculino são escassas ${ }^{19}$. No entanto, os resultados do presente estudo mostraram comportamento de risco para AN, entre os homens de ambos os cursos.

Resultado interessante foi visto em relação aos sintomas de AN com o decorrer do curso. Estudantes de nutrição tendem a reduzir o comportamento de risco para anorexia com o transcorrer do curso, enquanto alunos do curso de educação física apresentam comportamento contrário. Esse resultado pode estar relacionado ao fato de que com o passar dos anos, os alunos de nutrição adquirem conhecimentos mais amplos sobre alimentação e tendem a reduzir os riscos de transtornos e os alunos de educação física passam, cada vez mais, a se preocupar com a forma corporal.

A imagem corporal é a maneira pela qual o corpo se apresenta para si próprio. A indústria cultural, pelos meios de comunicação, encarrega-se de criar desejos e reforçar imagens padronizando corpos $^{20}$.

No presente estudo, ao avaliar a imagem corporal, houve tendência do curso de educação física apresentar proporções mais elevadas de insatisfação corporal (78,2\%), quando comparado ao curso de nutrição (75,8\%). Essa insatisfação corporal foi vista, especialmente, entre os alunos do sexo masculino da educação física. 
Estudo de Russo ${ }^{20}$, ao analisar a percepção corporal de estudantes educação física, de ambos os sexos, de uma cidade de São Paulo, concluiu que há diferenças em relação à insatisfação corporal entre os sexos, e que não somente o sexo feminino apresenta distúrbios estimulados pela cultura, como também os homens estão apresentando preocupação excessiva em ficar "forte" a todo custo. Isso explica os resultados encontrados no presente estudo, no qual estudantes do curso de educação física apresentaram maior desejo em aumentar de peso. Já nos estudantes de nutrição, a proporção de alunos com desejo de ser "normais" foi maior $(76,5 \%)$ do que entre os alunos de educação física $(48,7 \%)(p<0,05)$.

$\mathrm{Na} \mathrm{AN}$, há preocupação excessiva com o peso e a forma corporal, e a imagem corporal é o fator de risco para o desenvolvimento de TA ${ }^{21}$. Entretanto, no presente trabaIho, não houve associação entre insatisfação corporal e presença de comportamento anoréxico. São necessários mais estudos nessa área, a fim de se obter maiores esclarecimentos sobre esse distúrbio que vêm aumentando em nossa sociedade.

O presente estudo demonstrou que o curso de nutrição apresentou tendência maior de comportamento de risco para o desenvolvimento de AN, em relação ao curso de educação física, com predominância no sexo feminino.

Além disso, os resultados encontrados evidenciaram proporções elevadas de insatisfação com a imagem corporal, em ambos os cursos e desejo de alterá-la para adequar-se aos padrões sociais vigentes. Estes resultados são preocupantes, tendo em vista que são futuros professores de educação física e nutricionistas que possuem papel fundamental na detecção e, no caso do nutricionista, no manejo destes distúrbios.

\section{CONCLUSÃO}

É imprescindível que continuem as pesquisas no sentido de mais bem entender os distúrbios alimentares e sua ligação com os profissionais da área da saúde, uma vez que estes estão sujeitos a pressões da sociedade.

\section{REFERÊNCIAS}

1. Magalhães VC, Mendonça GAS. Transtornos alimentares em universitárias: estudo de confiabilidade da versão brasileira de questionários autopreenchíveis. Rev Bras Epidemiol. 2005:(8)3:236-45.

2. Fiates GMR, Salles RK. Fatores de risco para o desenvolvimento de distúrbios alimentares: um estudo em universitárias. Rev Nutrição. 2001;14(Suppl 0):3-6.

3. Assunção SSM, Cordas TA, Araújo LASB. Atividade física e transtornos alimentares. Rev Psiquiatr Clin. 2002;19(1):4-13.

4. Vilela, JEM, Lamounier JA, Filho MAD, Neto JRB, Horta GM. Transtornos alimentares em escolares. J Pediatria. 2004;(80)1:49-54.

5. Andrade A, Bosi MLM. Mídia e subjetividade no comportamento alimentar feminino. Rev Nutrição. 2003;(16)1:117-25.

6. Morgan CM, Vecchiatti IR, Negrão AB. Etiologia dos transtornos alimentares: aspectos biológicos, psicológicos e sócio-culturais. Rev Bras Psiquiatr. 2002;24(Suppl 3):18-23.

7. Oliveira FP, Bosi MLM, Vigário OS, Vieira RS. Comportamento a limentar e imagem corporal em atletas. Revista Brasileira de Medicina do Esporte. 2003;(9)6:348-56.

8. Melin P, Araújo AM. Transtornos alimentares em homens: um desafio diagnóstico. Rev Bras Psiquiatr. 2002;24(Suppl 3):73-6.

9. Assunção SSM. Dismorfia muscular. Rev Bras Psiquiatr. 2002;24(Suppl 3):80-4.

10. Garner DM, Olmsted MP, Boher Y, Garfikel PE. The Eating Attitudes Test: psychometric features and clinical correlates. Physiological Medicine. 1982;(12):871-8.

11. Nunes MA, Bagatini LF, Abuchaim AL, Kunz A, Ramos D, Silva JA, et al. Distúrbios da conduta alimentar: considerações sobre 0 teste de atitudes alimentares (EAT). Rev ABP-APAL. 1994; (16)1:7-10

12. Madrigal HF, Estévez Jl, Martínez MAG, Kearney J, Gibney M, Martínez JAH. Percepción de la imagem corporal como aproximación cualitativa al estado de nutrición. Salud Publica Mex. 1999;(41)6:479-86.

13. Pinzon V, Nogueira FC. Epidemiologia, curso e evolução dos transtornos. Rev Psiquiatr Clin. 2004;(31)4:158-60

14. Vitolo MR, Bortolini GA, Horta RL. Prevalência de compulsão alimentar entre universitárias de diferentes áreas de estudo. Rev Psiquiatria. 2006;(28)1:20-6.

15. Bosi MLM, Luiz RR, Morgado CMC, Costa MLS, Carvalho RJ. Autopercepção da imagem corporal entre estudantes de nutrição no Rio de Janeiro. J Brasileiro Psiquiatria. 2006;(55)2:108-13.

16. Furlan AC. Anorexia em alunas de educação física. Rev Mackenzie Educ Fis Esp. 2006;5:E23-30.

17. Faria P, Shinohara H. Transtornos alimentares. Rev Interação. 1998;(2)2:51-73.

18. Espíndola CR, Blay SL. Bulimia e transtorno da compulsão alimentar periódica: revisão sistemática e metassíntese. Rev Psiquiatria. 2006;(28)3:265-75.

19. Ribeiro KMN, Busse SR. Anorexia nervosa em adolescentes do sexo masculino: relato de casos. Rev Neuropsiquiatria. 2002;10(2):53-5.

20. Russo R. Imagem corporal: constrição através da cultura do belo. Movimento e Percepção. 2005;(5)6:80-90.

21. Philippi ST, Alvarenga M. Transtornos alimentares: uma visão nutricional. São Paulo: Manole; 2004. 